\title{
PI3K/mTOR/ALK-1/DNA-PK Inhibitor P7170
}

National Cancer Institute

\section{Source}

National Cancer Institute. PI3K/mTOR/ALK-1/DNA-PK Inhibitor P7170. NCI Thesaurus.

Code C104292.

An orally bioavailable inhibitor of phosphoinositide 3-kinase (PI3K), mammalian target of rapamycin (mTOR), activin receptor-like kinase 1 (ALK-1) and DNA-dependent protein kinase (DNA-PK), with potential anti-ang iogenic and antineoplastic activities. Upon oral administration, PI3K/mT OR/ALK-1/DNA-PK inhibitor P7170 inhibits the activity of all four kinases. This prevents PI3K/mTOR and ALK-1-mediated signaling pathways and may lead to the inhibition of cancer cell growth in PI3K/mT OR-overexpressing tumor cells and angiogenesis in ALK-1-overexpressing endothelial cells. Also, by inhibiting DNA-PK, this agent inhibits the ability of tumor cells to repair damaged DNA. The PI3K/mT OR pathway is upregulated in a variety of tumors and plays an important role in regulating cancer cell proliferation, growth, and survival. ALK-1, a member of the transforming growth factor beta (T GF-b) type I receptor family, is overexpressed on endothelial cells in a variety of tumor types and increases endothelial cell proliferation and migration. DNA-PK is activated upon DNA damage and plays a key role in repairing double-stranded DNA breaks. 